PROCEEDINGS OF THE

AMERICAN MATHEMATICAL SOCIETY

Volume 131, Number 2, Pages 631-636

S 0002-9939(02)06834-X

Article electronically published on September 25, 2002

\title{
A LOWER BOUND FOR SUMS OF EIGENVALUES OF THE LAPLACIAN
}

\author{
ANTONIOS D. MELAS
}

(Communicated by Bennett Chow)

\begin{abstract}
Let $\lambda_{k}(\Omega)$ be the $k$ th Dirichlet eigenvalue of a bounded domain $\Omega$ in $\mathbb{R}^{n}$. According to Weyl's asymptotic formula we have

$$
\lambda_{k}(\Omega) \sim C_{n}(k / V(\Omega))^{2 / n} .
$$

The optimal in view of this asymptotic relation lower estimate for the sums $\sum_{j=1}^{k} \lambda_{j}(\Omega)$ has been proven by P.Li and S.T.Yau (Comm. Math. Phys. 88 (1983), 309-318). Here we will improve this estimate by adding to its righthand side a term of the order of $k$ that depends on the ratio of the volume to the moment of inertia of $\Omega$.
\end{abstract}

\section{INTRODUCTION}

Let $\Omega \subseteq \mathbb{R}^{n}$ be a bounded open set and let $0<\lambda_{1}(\Omega) \leq \lambda_{2}(\Omega) \leq \ldots$ denote the eigenvalues (repeated with multiplicity) of the Dirichlet Laplacian on $\Omega$, that is, of the eigenvalue problem

$$
\begin{aligned}
\Delta u+\lambda u & =0 \text { in } \Omega, \\
u & =0 \text { on } \partial \Omega .
\end{aligned}
$$

The asymptotic behavior of $\lambda_{k}(\Omega)$ as $k \rightarrow \infty$ relates to geometric properties of the open set $\Omega$. In fact Weyl's asymptotic formula asserts that

$$
\lambda_{k}(\Omega) \sim C_{n}\left(\frac{k}{V(\Omega)}\right)^{2 / n} \text { as } k \rightarrow \infty
$$

where $V(\Omega)$ is the volume of $\Omega$ and $C_{n}=(2 \pi)^{2} \omega_{n}^{-2 / n}$ with $\omega_{n}$ being the volume of the unit ball in $\mathbb{R}^{n}$. Pólya proved in 4 that the above asymptotic relation is in fact a one-sided inequality if $\Omega$ is a plane domain that tiles $\mathbb{R}^{2}$ (and his proof also works in $\mathbb{R}^{n}$ ) and he conjectured, for any domain $\Omega$ in $\mathbb{R}^{n}$, the inequality

$$
\lambda_{k}(\Omega) \geq C_{n}\left(\frac{k}{V(\Omega)}\right)^{2 / n}
$$

for all $k \geq 1$.

In this direction Lieb $\left[3\right.$ proved an inequality like (1.3) for any domain $\Omega$ in $\mathbb{R}^{n}$ but with a constant $\tilde{C}_{n}$ that differs from the constant $C_{n}$ by a factor.

Received by the editors August 28, 2001.

2000 Mathematics Subject Classification. Primary 58G25; Secondary 35P15, 58G05.

(C)2002 American Mathematical Society 
Then P.Li and S.T.Yau [2] proved that on the average (1.3) is true for any domain $\Omega$ in $\mathbb{R}^{n}$, that is,

$$
\sum_{j=1}^{k} \lambda_{j}(\Omega) \geq \frac{n C_{n}}{n+2} k^{\frac{n+2}{n}} V(\Omega)^{-\frac{2}{n}}
$$

which is sharp in view of Weyl's asymptotic formula. This also gives a lower bound for each individual eigenvalue, better than previously known and tending to be optimal as the dimension $n \rightarrow \infty$. This inequality was complemented by P.Kröger in [1] who gave an upper bound for the sums of the eigenvalues depending on geometric properties of $\Omega$ that have to do with the behavior of the volume of the $\varepsilon$-neigbourhoods of the boundary $\partial \Omega$. Using this he obtained close to optimal estimates for individual eigenvalues that however depend on these geometric assumptions.

Here we will obtain an improvement of the estimate (1.4). Let $I(\Omega)$ denote the "moment of inertia" of $\Omega$, that is,

$$
I(\Omega)=\min _{a \in \mathbb{R}^{n}} \int_{\not}|x-a|^{2} d x .
$$

Then we have the following.

Theorem 1. For any bounded open set $\Omega \subseteq \mathbb{R}^{n}$ and any $k \geq 1$ we have

$$
\sum_{j=1}^{k} \lambda_{j}(\Omega) \geq \frac{n C_{n}}{n+2} k^{\frac{n+2}{n}} V(\Omega)^{-\frac{2}{n}}+M_{n} k \frac{V(\Omega)}{I(\Omega)}
$$

where the constant $M_{n}$ depends only on the dimension.

In fact one may take $M_{n}=\frac{c}{n+2}, c$ being independent of $n$, as the proof will show. The proof will follow in part the argument of Li and Yau in [2].

\section{LOWER ESTIMATE FOR SUMS OF EIGENVALUES}

In this section we will prove Theorem 1.

By translating the open set $\Omega$ we may assume that

$$
I(\Omega)=\int_{\not}|x|^{2} d x .
$$

We now fix a $k \geq 1$ and let $u_{1}, \ldots, u_{k}$ denote an orthonormal set of eigenfunctions of (1.1) corresponding to the set of eigenvalues $\lambda_{1}(\Omega), \lambda_{2}(\Omega), \ldots, \lambda_{k}(\Omega)$. We consider the Fourier transform of each eigenfunction

$$
f_{j}(\xi)=\hat{u}_{j}(\xi)=(2 \pi)^{-n / 2} \int_{\Omega} u_{j}(x) e^{i x \cdot \xi} d x .
$$

Plancherel's Theorem implies that $f_{1}, \ldots, f_{k}$ is an orthonormal set in $\mathbb{R}^{n}$. Since $u_{1}, \ldots, u_{k}$ are also orthonormal in $L^{2}(\Omega)$, Bessel's inequality implies that for every $\xi \in \mathbb{R}^{n}$

$$
\sum_{j=1}^{k}\left|f_{j}(\xi)\right|^{2} \leq(2 \pi)^{-n} \int_{\Omega}\left|e^{i x \cdot \xi}\right|^{2} d x=(2 \pi)^{-n} V(\Omega)
$$


and since

$$
\nabla f_{j}(\xi)=(2 \pi)^{-n / 2} \int_{\Omega} i x u_{j}(x) e^{i x \cdot \xi} d x
$$

that

$$
\sum_{j=1}^{k}\left|\nabla f_{j}(\xi)\right|^{2} \leq(2 \pi)^{-n} \int_{\Omega}\left|i x e^{i x \cdot \xi}\right|^{2} d x=(2 \pi)^{-n} I(\Omega) .
$$

Since each $u_{j}$ vanishes on the boundary of $\Omega$ it is easy to see that (see [2])

$$
\int_{\mathbb{R}^{n}}|\xi|^{2}\left|f_{j}(\xi)\right|^{2} d \xi=\int_{\Omega}\left|\nabla u_{j}(x)\right|^{2} d x=\lambda_{j}(\Omega)
$$

for each $j$. Hence setting

$$
F(\xi)=\sum_{j=1}^{k}\left|f_{j}(\xi)\right|^{2}
$$

we have $0 \leq F(\xi) \leq(2 \pi)^{-n} V(\Omega)$,

$$
|\nabla F(\xi)| \leq 2\left(\sum_{j=1}^{k}\left|f_{j}(\xi)\right|^{2}\right)^{1 / 2}\left(\sum_{j=1}^{k}\left|\nabla f_{j}(\xi)\right|^{2}\right)^{1 / 2} \leq 2(2 \pi)^{-n} \sqrt{V(\Omega) I(\Omega)}
$$

for every $\xi \in \mathbb{R}^{n}$ and moreover

$$
\int_{\mathbb{R}^{n}} F(\xi) d \xi=k \text { and } \int_{\mathbb{R}^{n}}|\xi|^{2} F(\xi) d \xi=\sum_{j=1}^{k} \lambda_{j}(\Omega) .
$$

Now let $F^{*}(\xi)=\phi(|\xi|)$ denote the decreasing radial rearrangement of $F$ where we may assume (by approximating $F$ ) that the decreasing function $\phi:[0,+\infty) \rightarrow$ $\left[0,(2 \pi)^{-n} V(\Omega)\right]$ is absolutely continuous. Setting $\mu(t)=\left|\left\{F^{*}>t\right\}\right|=|\{F>t\}|$ the coarea formula implies that

$$
\mu(t)=\int_{t}^{(2 \pi)^{-n} V(\Omega)} \int_{\{F=s\}}|\nabla F|^{-1} d \sigma_{s} d s .
$$

Since $F^{*}$ is radial we have $\mu(\phi(s))=\left|\left\{F^{*}>\phi(s)\right\}\right|=\omega_{n} s^{n}$ and so differentiating we get $n \omega_{n} s^{n-1}=\mu^{\prime}(\phi(s)) \phi^{\prime}(s)$ for almost every $s$. But (2.8), (2.10) and the isoperimetric inequality imply that, with $\rho=2(2 \pi)^{-n} \sqrt{V(\Omega) I(\Omega)}$,

$$
\begin{aligned}
-\mu^{\prime}(\phi(s)) & =\int_{\{F=\phi(s)\}}|\nabla F|^{-1} d \sigma_{\phi(s)} \geq \rho^{-1} \operatorname{Vol}_{n-1}(\{F=\phi(s)\}) \\
& \geq \rho^{-1} n \omega_{n} s^{n-1}
\end{aligned}
$$

and so

$$
-\rho \leq \phi^{\prime}(s) \leq 0
$$

for almost every $s$.

Now (2.9) implies that

$$
k=\int_{\mathbb{R}^{n}} F(\xi) d \xi=\int_{\mathbb{R}^{n}} F^{*}(\xi) d \xi=n \omega_{n} \int_{0}^{\infty} s^{n-1} \phi(s) d s
$$


and

$$
\sum_{j=1}^{k} \lambda_{j}(\Omega)=\int_{\mathbb{R}^{n}}|\xi|^{2} F(\xi) d \xi \geq \int_{\mathbb{R}^{n}}|\xi|^{2} F^{*}(\xi) d \xi=n \omega_{n} \int_{0}^{\infty} s^{n+1} \phi(s) d s
$$

since $\xi \rightarrow|\xi|^{2}$ is radial and increasing.

To prove Theorem 1 we will also need the following lemma.

Lemma 1. Let $n \geq 1, \rho, A>0$ and $\psi:[0,+\infty) \rightarrow[0,+\infty)$ be decreasing (and absolutely continuous) such that

$$
-\rho \leq \psi^{\prime}(s) \leq 0
$$

and

$$
\int_{0}^{\infty} s^{n-1} \psi(s) d s=A
$$

Then

$$
\int_{0}^{\infty} s^{n+1} \psi(s) d s \geq \frac{1}{n+2}(n A)^{\frac{n+2}{n}} \psi(0)^{-\frac{2}{n}}+\frac{A \psi(0)^{2}}{6(n+2) \rho^{2}} .
$$

Proof. By considering the function $\alpha \psi(\beta t)$ for appropriate $\alpha, \beta>0$ we may assume that $\rho=1$ and $\psi(0)=1$. We also assume that $B=\int_{0}^{\infty} s^{n+1} \psi(s) d s<\infty$, otherwise there is nothing to prove, and so $\lim _{j \rightarrow \infty} T_{j}^{n+1} \psi\left(T_{j}\right)=0$ for some sequence $\left(T_{j}\right)$ with $T_{j} \rightarrow \infty$ as $j \rightarrow \infty$.

Let $h(s)=-\psi^{\prime}(s)$ for $s \geq 0$. Then $0 \leq h(s) \leq 1$ and $\int_{0}^{\infty} h(s) d s=\psi(0)=1$. Moreover integration by parts shows that

$$
\int_{0}^{\infty} s^{n} h(s) d s=n \int_{0}^{\infty} s^{n-1} \psi(s) d s=n A
$$

since $\lim _{j \rightarrow \infty} T_{j}^{n+1} \psi\left(T_{j}\right)=0$, and

$$
\int_{0}^{\infty} s^{n+2} h(s) d s=\lim _{T \rightarrow \infty}\left(-T^{n+2} \psi(T)+(n+2) \int_{0}^{T} s^{n+1} \psi(s) d s\right) \leq(n+2) B .
$$

Next let $a \geq 0$ be such that

$$
\int_{a}^{a+1} s^{n} d s=\int_{0}^{\infty} s^{n} h(s) d s=n A .
$$

Such an $a$ exists since by the same argument as in Lemma 1 of [2] one can easily show that the assumptions on $h$ imply $\int_{0}^{\infty} s^{n} h(s) d s \geq \int_{0}^{1} s^{n} d s$. Indeed this follows by integrating the inequality $\left(s^{n}-1\right)(h(s)-\xi(s)) \geq 0$ over $[0,+\infty)$ where $\xi$ is the characteristic function of the interval $[0,1]$. We also choose $\lambda, \mu \in \mathbb{R}$ such that the function

$$
q(s)=s^{n+2}-\lambda s^{n}+\mu
$$

satisfies $q(a)=q(a+1)=0$. Since the derivative $q^{\prime}(s)$ has at most one zero in $[0,+\infty)$ we conclude that $q(s)<0$ in $(a, a+1)$ and $q(s)>0$ in $[0,+\infty) \backslash(a, a+1)$ (and also $\lambda, \mu \geq 0)$. Thus letting $\chi(s)$ denote the characteristic function of the interval $[a, a+1]$, the assumptions on $h$ imply that

$$
q(s)(\chi(s)-h(s)) \leq 0 \text { on }[0,+\infty) .
$$


Integrating the inequality (2.22), taking into account the choice of $a$ and using (2.19) we have

$$
(n+2) B \geq \int_{0}^{\infty} s^{n+2} h(s) d s \geq \int_{a}^{a+1} s^{n+2} d s .
$$

To estimate the last integral we take $\tau>0$ to be chosen later and integrate the inequality

$$
n s^{n+2}-(n+2) \tau^{2} s^{n}+2 \tau^{n+2} \geq 2 \tau^{n}(s-\tau)^{2}
$$

(that can be proved by dividing the left-hand side by $\left.(s-\tau)^{2}\right)$ over $[a, a+1]$ to get, also using (2.23), that

$$
\begin{aligned}
n(n+2) B-(n+2) \tau^{2} n A+2 \tau^{n+2} & \geq 2 \tau^{n} \int_{a}^{a+1}(s-\tau)^{2} d s \geq \\
& \geq 2 \tau^{n} \int_{-1 / 2}^{1 / 2} t^{2} d t=\frac{\tau^{n}}{6} .
\end{aligned}
$$

Now choosing $\tau=(n A)^{1 / n}$ we get

$$
B \geq \frac{1}{n+2}(n A)^{\frac{n+2}{n}}+\frac{A}{6(n+2)}
$$

and this completes the proof of the Lemma.

To complete the proof of Theorem 1 we apply Lemma 1 to the function $\phi$ with $A=\left(n \omega_{n}\right)^{-1} k, \rho=2(2 \pi)^{-n} \sqrt{V(\Omega) I(\Omega)}$ and get in view of (2.14) that

$$
\sum_{j=1}^{k} \lambda_{j}(\Omega) \geq \frac{n}{n+2} \omega_{n}^{-\frac{2}{n}} k^{\frac{n+2}{n}} \phi(0)^{-\frac{2}{n}}+\frac{c k \phi(0)^{2}}{(n+2) \rho^{2}}
$$

where $c$ is any constant such that $0<c<\frac{1}{6}$.

Now observe that $0<\phi(0) \leq(2 \pi)^{-n} V(\Omega)$ and that if $R$ is such that $\omega_{n} R^{n}=$ $V(\Omega)$, then $I(\Omega) \geq \int_{B(R)}|x|^{2} d x=\frac{n \omega_{n} R^{n+2}}{n+2}$ and so

$$
\rho \geq 2(2 \pi)^{-n} \sqrt{\frac{n}{n+2} \omega_{n}^{-\frac{2}{n}} V(\Omega)^{\frac{n+2}{n}+1}} \geq(2 \pi)^{-n} \omega_{n}^{-\frac{1}{n}} V(\Omega)^{\frac{n+1}{n}} .
$$

On the other hand the function $g(t)=\frac{n}{n+2} \omega_{n}^{-\frac{2}{n}} k^{\frac{n+2}{n}} t^{-\frac{2}{n}}+\frac{c k t^{2}}{(n+2) \rho^{2}}$ would be decreasing on $\left(0,(2 \pi)^{-n} V(\Omega)\right]$ if $g^{\prime}\left((2 \pi)^{-n} V(\Omega)\right) \leq 0$ which in view of (2.28) and since $k \geq 1$ will be satisfied if

$$
c<(2 \pi)^{2} \omega_{n}^{-\frac{4}{n}} .
$$

It is easy to see that we can choose $c$ independent of $n$ that satisfies (2.29). Then we can replace $\phi(0)$ by $(2 \pi)^{-n} V(\Omega)$ in (2.27) which gives inequality (1.6) and so completes the proof of Theorem 1. 


\section{REFERENCES}

[1] P.Kröger: Estimates for sums of Eigenvalues of the Laplacian, Jour. Funct. Anal. 126 (1994), 217-227. MR 95j:58173

[2] P.Li, S.T.Yau: On the Schrödinger equation and the eigenvalue problem, Comm. Math. Phys. 88 (1983), 309-318. MR 84k:58225

[3] E.Lieb: The number of bound states of one-body Schröndinger operators and the Weyl problem, Proc. Sym. Pure Math. 36 (1980), 241-252. MR 82i:35134

[4] G.Pólya: On the eigenvalues of vibrating membranes, Proc. London Math. Soc. (3) 11 (1961), 419-433. MR 23:B2256

[5] B.Simon: Weak trace ideals and the number of bound states of Schrödinger operators, Trans. Amer. Math. Soc. 224 (1976), 367-380. MR 54:11109

[6] R.S.Strichartz: Estimates for sums of eigenvalues for domains in homogeneous spaces, Jour. Funct. Anal. 137 (1996), 152-190. MR 97g:58172

Department of Mathematics, University of Athens, Panepistimiopolis 15784, Athens, GREece

E-mail address: amelas@math.uoa.gr 